\title{
On the Calibration and Performance of RSS-based Localization Methods
}

\author{
B.J.Dil ${ }^{1}$, P.J.M.Havinga ${ }^{2}$ \\ Pervasive Systems, University of Twente \\ Zilverling building, 7522 NB Enschede, The Netherlands \\ ${ }^{1}$ B.J.Dil@utwente.nl \\ ${ }^{2}$ P.J.M.Havinga@ewi.utwente.nl \\ Ambient Systems \\ Colosseum 15d, 7521 PV Enschede, The Netherlands \\ ${ }^{1}$ Bram@ambient-systems .net
}

\begin{abstract}
This paper analyzes the performance of several Received Signal Strength (RSS) based localization methods as a function of the calibration effort, hence as a function of deployment and maintenance costs. The deployment and maintenance costs determine the scalability and thus the applicability of a localization algorithm, and this is still a topic of research. This paper analyzes and compares the best available localization algorithms of the following localization methods: fingerprinting-, range- and proximity-based localization. An extensive amount of RSS measurements, performed in a realistic indoor environment show that range-based algorithms outperform fingerprintingand proximity-based localization algorithms when there is a limited amount of calibration measurements available. In that case, range-based algorithms have $\sim 30 \%$ smaller errors, $\sim 1.3$ meter compared to $\sim 1.9$ meter. Our measurements show that fingerprinting-based algorithms approximate the performance of range-based algorithms as the number of calibration measurements increases from 1 to 80 .
\end{abstract}

\section{INTRODUCTION}

This paper focuses on localization in wireless networks. Localization in these networks describes the process of obtaining a physical location in an automated manner using wireless communication. Many wireless network applications rely on location information to perform their tasks. Locations provide context to the measured data (e.g. like measuring temperature); localization can be a stand-alone application (e.g. inventory tracking in a distribution center) or it provides support to the network service (e.g. routing). Today, such applications have evolved into real-time location systems (RTLS) using a wide range of wireless technologies. Many of these localization applications are based on Received Signal Strength (RSS) measurements, as RSS information is obtained without additional hardware and energy costs. Other localization systems use techniques like Time Difference Of Arrival (TDOA), Time Of Flight (TOF), Ultra Wide Band (UWB) and Angle Of Arrival (AOA). In general, these techniques are more accurate than RSS-based localization but require specialized hardware, more processing, more communication and thus more energy ([16]).

This paper focuses on RSS-based localization. It deals with the required effort of several RSS-based localization methods to properly account for the influence of the environment both locally and over time versus the localization performance. Several practical examples that influence the signal strength are reflections, obstacles, temperature and humidity ([20]). RSS-based localization methods make use of propagation models to account for these influences. These propagation models differ in the calibration efforts they require to produce a certain accuracy. We distinguish the following calibration costs (as in [12]):

- Deployment costs represent the required time and effort to calibrate the propagation model before localization.

- Maintenance costs represent the required time and effort to update the propagation model, as most environments change over time ([4]).

Propagation models that require an extensive amount of calibration measurements have high deployment and maintenance costs, as the deployment and maintenance costs determine the scalability and applicability of localization algorithms. Moreover, the deployment and maintenance costs increase the implementation and network complexity, communication overhead and required hardware ([12]). To our knowledge, this is the first paper that analyzes the performance of several RSS-based localization methods as a function of the number of calibration measurements. We distinguish the following RSS-based localization methods, i.e. fingerprinting-, rangeand proximity-based localization. Existing work on calibration effort versus the localization performance mainly focuses on one localization method, as in [22].

Figure 1 shows how existing RSS-based localization methods calibrate the propagation model. The calibration takes place in the "Calibrate propagation model" phase. In this phase, the propagation model is calibrated on the basis of the calibration measurements. During the second phase, the "Localization" phase, the position of the node is estimated, both on the basis of the localization measurements as well as on the calibrated propagation model. Hence, existing RSS-based localization algorithms assume that the propagation model is calibrated before the "Localization" phase. In this paper, we separately 


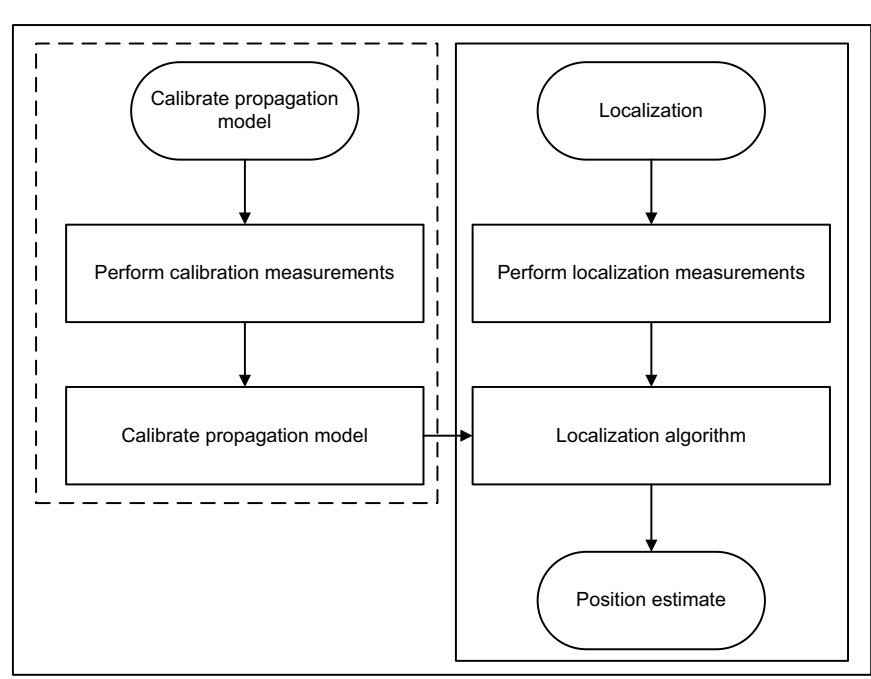

Fig. 1. Existing Localization Approach

analyze and compare the calibration and localization phase of several RSS-based localization methods by performing an extensive amount of measurements. We performed two rounds of RSS measurements in the same set-up, one measurement round to calibrate the propagation model and one measurement round to evaluate the performance. We use these measurements to quantify the influence of relatively small deviations (centimeters) in the measurement locations on the RSS measurements. The main contributions of this work are:

- Quantify the influence of relatively small deviations (centimeters) in the measurement locations on the RSS. These measurements show that on average the resulting RSS may vary more than a factor of two $(3 \mathrm{dBm})$.

- Evaluate the performance of three RSS-based localization methods as a function of the number of calibration measurements. The measurements show that range-based algorithms outperform fingerprinting- and proximity-based localization algorithms when there is a limited amount of calibration measurements available. In that case, rangebased algorithms have $\sim 30 \%$ smaller errors, from $\sim 1.9$ to $\sim 1.3$ meter. Our measurements show that fingerprinting-based algorithms approximate the performance of range-based algorithms when the number of calibration measurements increases from 1 to 80 .

This paper is organized as follows. After a short overview of existing RSS-based localization methods in Section II, we present the localization and measurement set-up in Section III. Section IV analyzes the calibration of the propagation models of the localization methods. Section V analyzes the influence of the number of calibration measurements on the performance of existing RSS-based localization methods. Section VI summarizes the results.

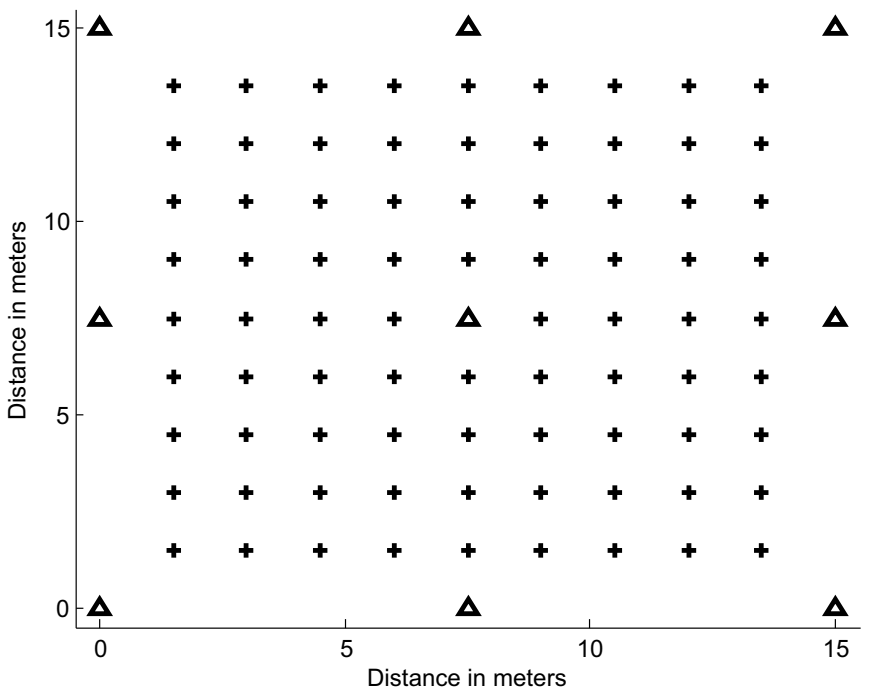

Fig. 2. Measurement set-up

\section{RELATED WORK}

This section provides a short overview of existing RSSbased localization methods. These localization methods are fingerprinting, range-based, range-free and proximity-based localization.

\section{- Fingerprinting:}

Many RSS-based localization systems make use of fingerprinting ([18] and [25]), first proposed by [3]. In the calibration phase, the signal strength is measured from static infrastructure nodes at several locations. The measurements, taken at a particular position, represent the fingerprint of that particular position. The localization area is thus divided into a large set of positions and measured fingerprints. The stored fingerprints represent the parameters that are calibrated in the calibration phase. The localization phase consists of finding the closest match with the localization measurements in the database of fingerprints. Fingerprinting achieves a relatively high accuracy in static indoor environments, as it copes with static sources of noise such as walls that are common in indoor environments. Although much has been done in the field of fingerprinting-based localization, [3] still delivers similar results as the newest fingerprinting algorithms when the number of calibration measurements is high enough ([25]). We evaluate the performance with the Weighted K-Nearest Neighbor algorithm (WKNN, [18]) using linear Delaunay Triangulation interpolation ([13]). This algorithm provides the best performance when the number of calibration measurements is limited (e.g. [18] and [25]).

\section{- Range-based Localization:}

Range-based localization algorithms assume that the signal strength decay over distance follows a distribution that is known a priori. This distribution is used for converting one or several signal strength measurements 


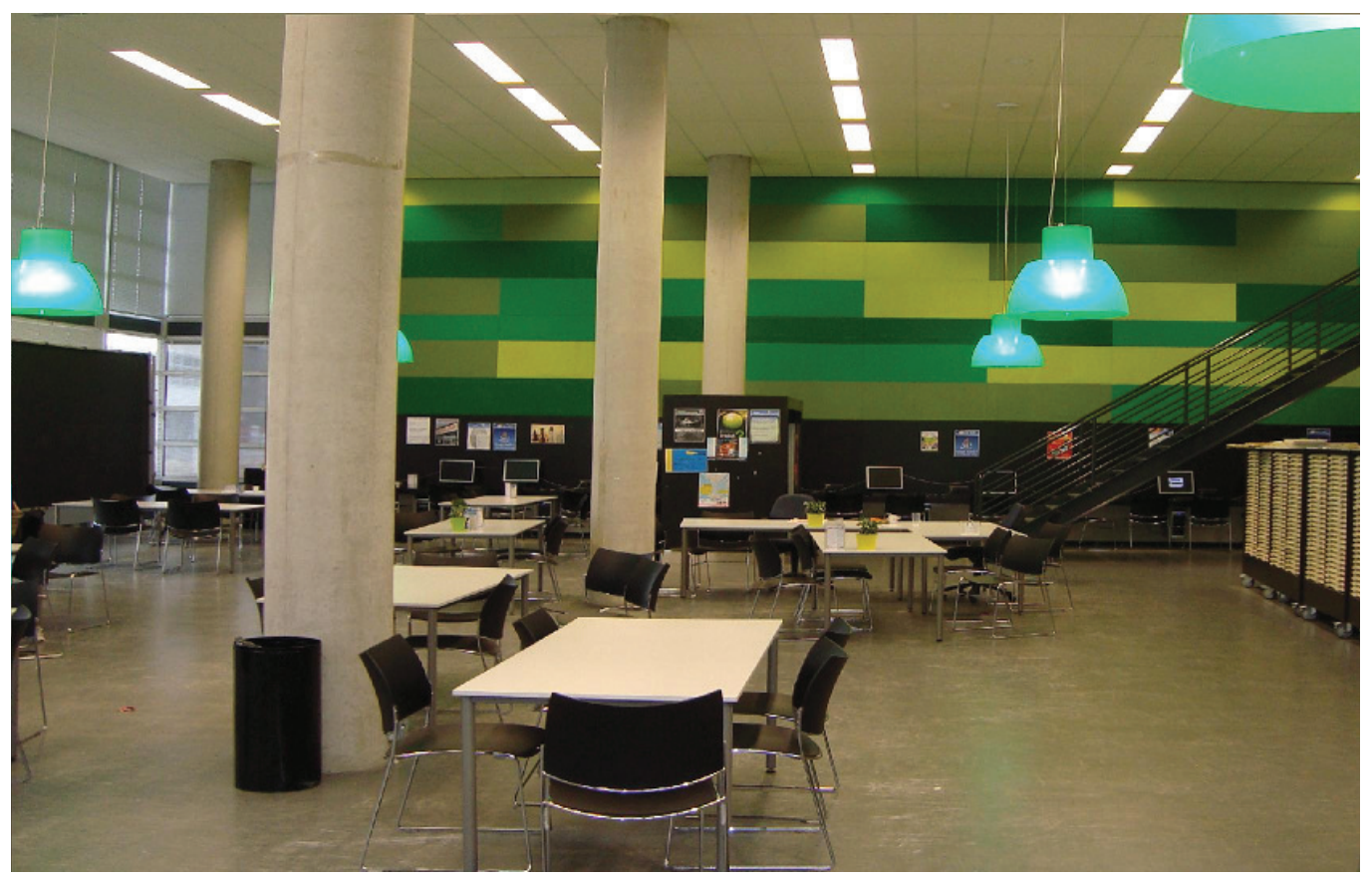

Fig. 3. Measurement environment

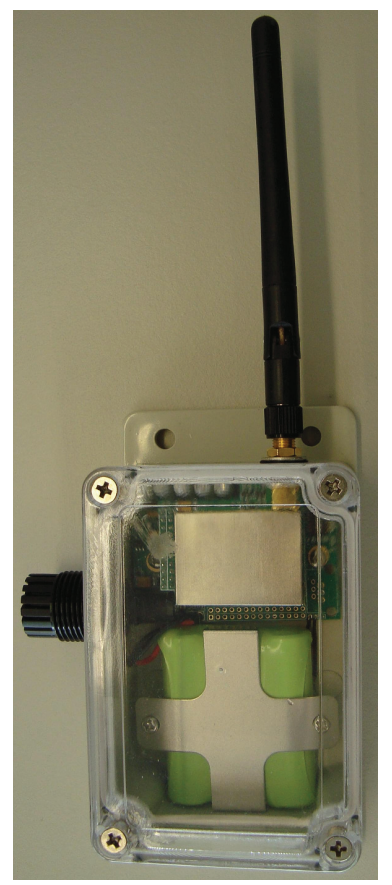

Fig. 4. CC2430 radio into distance estimates. These distributions often include several parameters that try to account for the influence of the environment which are calibrated in the calibration phase (e.g. [5], [17], [19] and [24]). In this paper, we evaluate the performance of the Maximum Likelihood Estimator based on the Log-Normal Shadowing model ([5]). To our knowledge, [5] provides the best results given the Log-Normal Shadowing model.

\section{- Range-free Localization:}

Range-free localization algorithms assume that the transmission range or the deployment distribution is known before the localization phase (e.g. [6], [9] and [26]). The transmission range and/or the deployment distribution are considered as parameters that are calibrated before localization. The performance of these algorithms depends on the difference between the expected and real values of the transmission range and/or deployment distribution. Radio connectivity information turns signal strength measurements into discrete quantities. This quantization decreases the localization accuracy compared to using analogue signal strength measurements ([16]). Therefore, we do not consider range-free localization algorithms in this paper.

\section{- Proximity-based Localization:}

Proximity based localization algorithms assume that the signal strength decays inversely proportionally with distance ([8], [14] and [15]). The main difference with range-based algorithms is that proximity-based localization only uses the order of RSS measurements instead of converting signal strength to distance estimates. The advantage of proximity based localization algorithms is that they do not require a calibration phase. In this paper, we evaluate the performance of ecolocation ([15]). To our knowledge, ecolocation provides the best performance of the RSS-based proximity localization algorithms ([15]). Moreover, its performance does not depend on RSS measurements between reference nodes (as in [8] and [14]).

\section{Localization AND MeAsurement Set-uP}

This section first provides a formal description of the localization problem. After the problem formulation, this section provides a description of the measurement set-up. Consider a wireless network that consists of $N$ reference nodes and $M$ blind nodes:

- Reference nodes know their position in advance.

- Blind nodes do not know their location in advance and are subject to localization.

This paper addresses the problem of positioning blind nodes using signal strength measurements from several reference nodes. We do not evaluate signal strength measurements between blind nodes (like in [5] and [17]).

Figure 2 shows the measurement set-up used throughout this paper. Here the nine triangles represent the reference node locations; the crosses represent the blind node locations. The measurements were conducted in a $15 \times 15$ meter indoor study environment with ten CC2430 radio's ([28]). We used nine CC2430 radio's as reference nodes; these reference nodes were static during and between the measurement rounds (triangles). We used one CC2430 radio as blind node; this blind node measured the RSS at 80 different locations (crosses) relative to the reference nodes. The blind node measured 100 consecutive 


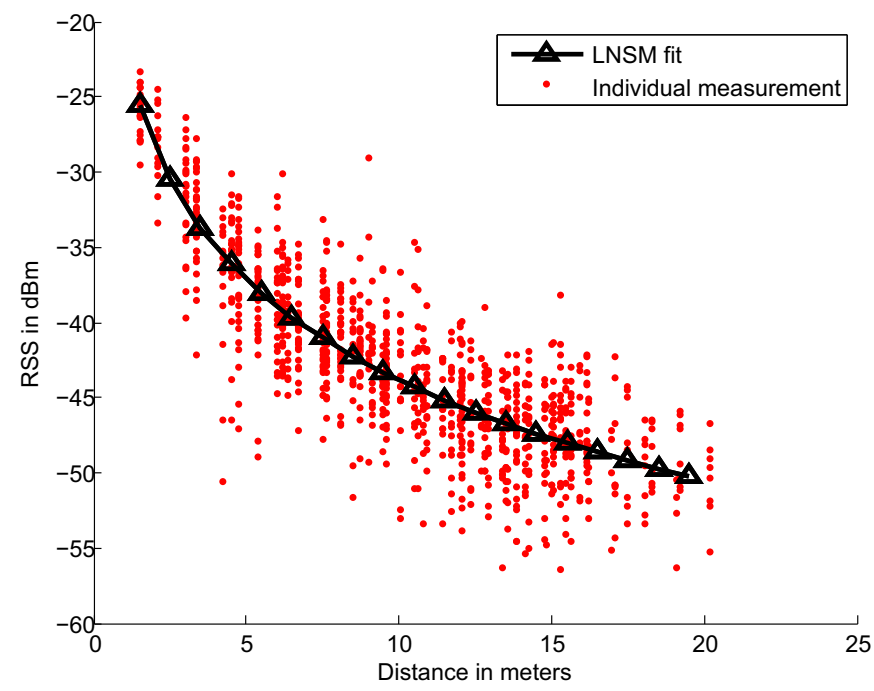

Fig. 5. RSS over distance distribution

\begin{tabular}{|l|l|l|}
\hline Approach & Calibration measurements & Localization measurements \\
\hline Ideal & Calibration measurements & Calibration measurements \\
Realistic & Calibration mesaurements & Localization measurements \\
\hline
\end{tabular}

TABLE I

PERFORMANCE EVALUATION

RSS measurements per frequency over a total of 38 frequencies in a bandwidth of $2408 \ldots 2480 \mathrm{MHz}$. Figure 3 shows the indoor environment and Figure 4 shows one CC2430 radio with casing. The nodes all had a widely used "omnidirectional" dipole antenna with a vertical orientation. We performed two sets of measurement rounds on each location on different days. This means that "equal" measurement locations differ in the order of centimeters. Hence, the difference in RSS over the measurement rounds represents the influence on the RSS of relatively small deviations in the measured locations. Throughout this paper, we consider measurement round one as the calibration measurements and measurement round two as the localization measurements. The radio's were all placed at the same height at two meters in order to minimize noise (e.g. [20]). All individual RSS measurements were sent to a computer and logged for post-processing. The conditions during the measurements were static (temperature, humidity, no moving objects). Therefore, we consider this environment as a static environment.

\section{CAlibration Propagation Model}

This section describes the calibration of the propagation models of the range-based and fingerprinting-based localization methods. The proximity-based localization method does not require any calibration measurements (see Section II), and is not included in this section. Throughout this paper, we distinguish two approaches to evaluate the performance:

- The ideal approach uses the calibration measurements

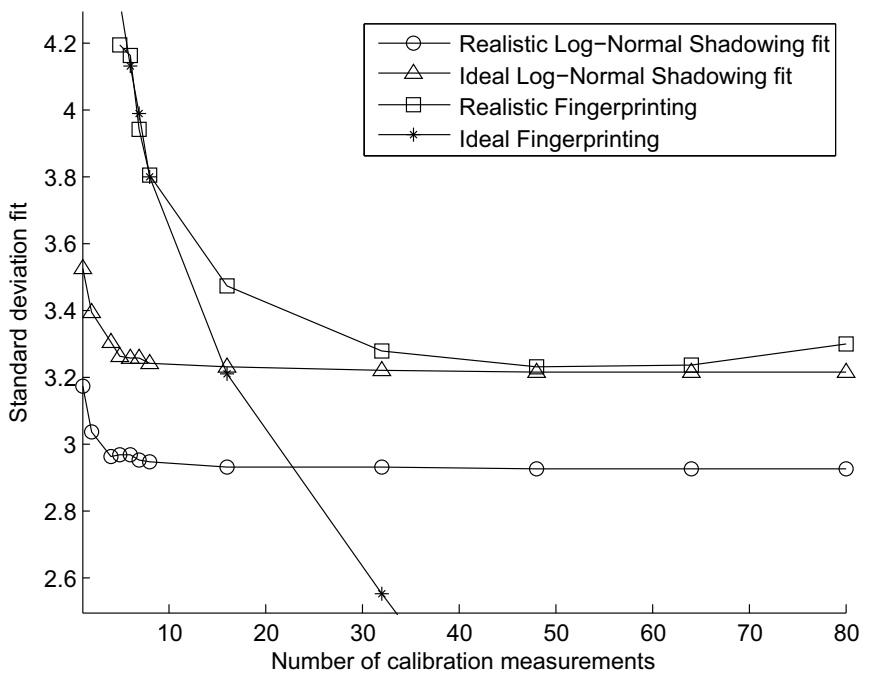

Fig. 6. Calibration accuracy

for both calibrating the propagation model and evaluating the performance. This performance evaluation approach provides an indication of the optimal localization performance, because the same measurements are used for calibrating and evaluating the localization algorithm.

- The realistic approach uses the calibration measurements to calibrate the propagation model and uses the localization measurements to evaluate the performance. This performance evaluation approach provides a more realistic indication of the performance, with the conditions during the calibration and measurements being essentially similar.

\section{A. Calibration of Range-based Method}

[5] uses the Log-Normal Shadowing Model (LNSM) for describing the signal strength over distance decay. This model is widely used by RSS-based localization algorithms (e.g. [17] and [19]) and has shown to be a reasonable representation of reality ([2]). This model assumes that the received signal strength follows a log-normal distribution ([1]):

$$
P_{d}=P_{d_{0}}-10 \cdot n \cdot \log _{10}\left(\frac{d}{d_{0}}\right)+X_{\sigma}
$$

Here:

- $P_{d}$ represents the received signal strength in $\mathrm{dBm}$ at distance $d$.

- $P_{d_{0}}$ represents the received signal strength in $d B m$ at reference distance $d_{0}$. In general distance $d_{0}$ is relatively small. For simplicity, we assume that distance $d_{0}$ is 1 meter (see [2]). Throughout this paper, we refer to this variable by "Reference RSS".

- $n$ represents the Path Loss Exponent (PLE). The path loss exponent represents the rate at which the path loss increases with distance.

- $\sigma_{d B m}$ represents the standard deviation of the received signal strength due to shadowing effects and is invariant 

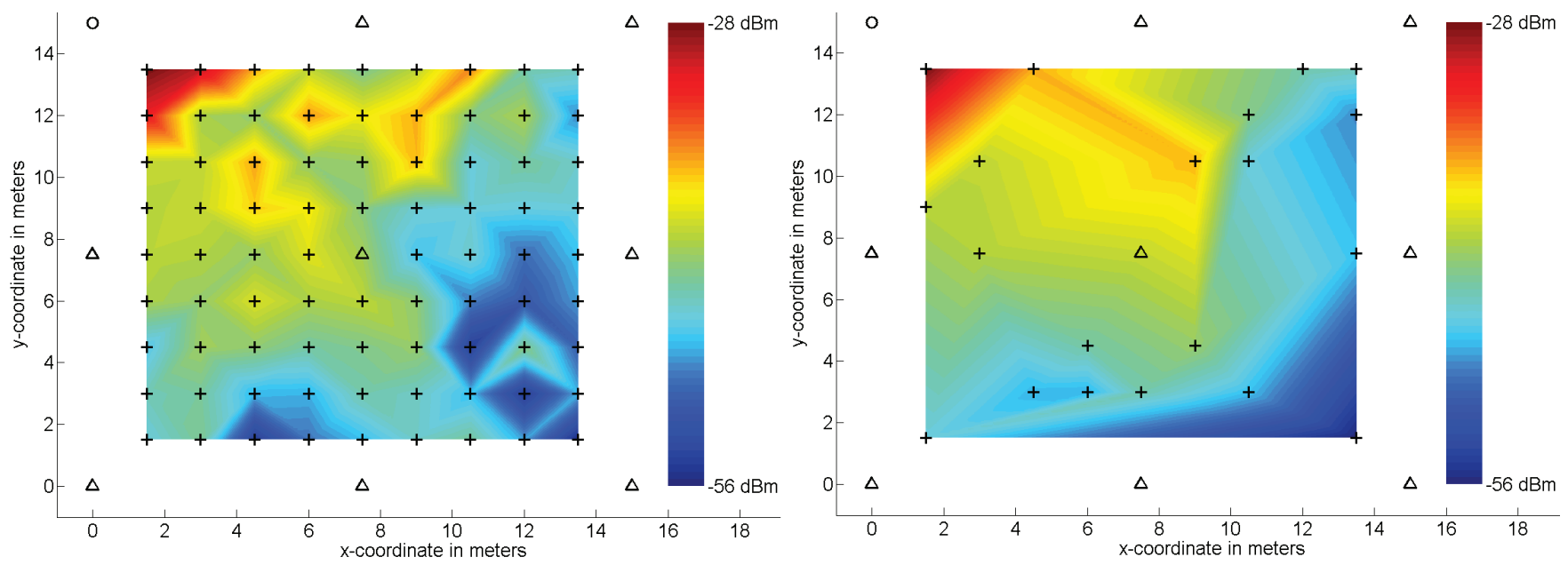

Fig. 7. RSS over surface distribution estimated by all calibration measurements Fig. 8. RSS over surface distribution estimated by 20 calibration measurements

with the distance ([2]). $X_{\sigma}$ follows a zero-mean normal distribution with standard deviation $\sigma_{d B m}$ :

$$
X_{\sigma} \sim N\left(0, \sigma_{d B m}^{2}\right)
$$

$\sigma_{d B m}$ is also an indication of how well the Log-Normal Shadowing Model fits the RSS measurements as it is equal to the standard deviation of the residuals.

Even though the model is widely accepted and has shown to be useful, it is important to note that it has limitations. Three major sources of error are multipath effects, shadowing ([1]) and hardware inaccuracies ([7] and [23]). The multipath effect is usually minimized by performing RSS measurements over a large frequency bandwidth. The remaining errors are caused by the attenuation of the signal due to obstructions (shadowing). In our experimental set-up, we used line-ofsight measurements to ensure that the major sources of error would be multipath effects and hardware inaccuracies. Figure 5 shows the Log-Normal Shadowing Model fitting the calibration measurements minimizing the squared residuals. The red dots represent individual RSS measurements. We distinguish two best fits, namely:

- The Log-Normal Shadowing Model that fits the calibration measurements (see Figure 5). The parameter values of this fit are: $\left\{P_{d_{0}}=-21.2 \mathrm{dBm}, n=2.29\right.$, $\left.\sigma_{d B m}=3.16 \mathrm{dBm}\right\}$.

- The Log-Normal Shadowing Model that fits the localization measurements (not shown in Figure 5). The parameter values of this fit are: $\left\{P_{d_{0}}=-22.4 \mathrm{dBm}, n=2.15\right.$, $\left.\sigma_{d B m}=2.9 \mathrm{dBm}\right\}$.

The parameter values show that the Log-Normal Shadowing Model of the calibration and localization measurements are approximately similar. Moreover, the individual fits clearly show that even though we performed RSS measurements over a frequency bandwidth of $74 \mathrm{MHz}$, multipath effects and hardware inaccuracies are still significant sources of error.

Figure 6 shows the accuracy of the estimated Log-Normal Shadowing Model by plotting the standard deviation of the residuals $\left(\sigma_{d B m}\right)$ as a function of the number of calibration measurements. We define one calibration measurement as the RSS measurements performed on one measured location (as defined in Section III). Moreover, the standard deviation of the residuals per number of calibration measurements is the mean over 50 runs. Per run we randomly pick measured locations equal to the number of calibration measurements. Note that we use the calibration measurements for calculating the parameters of the Log-Normal Shadowing model and that we use the localization measurements for calculating the residuals.

Figure 6 distinguishes two Log-Normal Shadowing fits using the performance evaluation approaches described as in Table I. This figure shows that:

- The accuracy of the fits increases marginally with an increasing number of calibration measurements.

- The "Realistic" fit has a smaller standard deviation than the "Ideal" fit. This is because the Log-Normal Shadowing model parameter settings of the calibration and localization measurements are approximately similar and the "Ideal" fit of the localization measurements has a lower standard deviation $\left(\sigma_{d B m}=2.9\right.$ in comparison with $\sigma_{d B m}=3.2$ ).

Note that the accuracy of the estimated path loss exponent $(n)$, reference RSS $\left(P_{d_{0}}\right)$ and shadowing component $\left(X_{\sigma}\right)$ can be estimated by using existing formulas within the field of linear regression. These formulas show that the calibration accuracy depends on the shadowing component, the number of calibration measurements and the measured locations relative to the reference nodes.

\section{B. Calibration of Fingerprinting-based Method}

In this section, we analyze the calibration accuracy of the WKNN algorithm using linear Delaunay Triangulation interpolation as a function of the number of calibration measurements. We use linear Delaunay Triangulation interpolation in order to estimate the RSS over surface distribution of 
the calibration measurements. Figure 7 and 8 show the RSS over surface distribution of one reference node given all and 20 calibration measurements. Here the circle represents the transmitting reference node; the triangles represent the non-transmitting reference nodes; the crosses represent the evaluated calibration measurement locations (see Section III). Note that Figure 7 and 8 clearly show the difference between using all and 20 calibration measurements. Moreover, linear Delaunay Triangulation interpolation requires that the interpolated fingerprints are within the convex hull of the calibration measurement locations. Therefore, the calibration measurement locations set constraints on the localization surface, which is also the case with other fingerprinting localization algorithms (e.g. [3]).

Figure 6 shows the accuracy of the estimated RSS using fingerprinting with linear Delaunay Triangulation interpolation as a function of the number of calibration measurements. We distinguish the same fits and use the same method as in Section IV-A. Figure 6 shows that:

- Fingerprinting requires significantly more calibration measurements than the Log-Normal Shadowing model. The accuracy of the "Realistic Fingerprinting" fit stabilizes with 32 or more measurements.

- The difference between the ideal and realistic fingerprinting fit is significantly larger than with the Log-Normal Shadowing model. This is understandable because fingerprinting uses the exact measurements for calibration, while the Log-Normal Shadowing fits the measurements.

\section{Localization Performance}

In this section we evaluate the performance of several RSS-based localization algorithms by performing an extensive amount of RSS measurements. Moreover, this section analyzes the localization performance as a function of the number of calibration measurements. We evaluate these algorithms in the set-up described in Section III, and we use the parameter values for the propagation models calculated in Section IV. We use the same performance evaluation approaches used in Section IV and described by Table I. This section compares the following RSS-based localization algorithms:

- Proximity-based localization algorithm: ECOLOCATION ([15], abbreviated by ECO).

- Range-based localization algorithm: Maximum Likelihood Estimator ([5]), abbreviated by MLE).

- Fingerprinting: WKNN ([18]) using linear Delaunay Triangulation interpolation ([13], abbreviated by RAD).

RAD put constraints on the position estimate, which increases the performance. Therefore, we also put the same constraints on the other localization algorithms in order to make a fair comparison. Note that we picked one localization algorithm per localization method described in Section II in order to characterize the performance per localization method.

\section{A. Number of Calibration Measurements}

Figure 9 shows the performance of the three localization algorithms as a function of the number of calibration mea- surements. Figure 9 shows that the "Realistic" performance of:

- ECO is independent of the number of calibration measurements. This is because ECO does not require/use calibration measurements.

- MLE provides marginally better performance as the number of calibration measurements increases (from $\sim 1.29$ to $\sim 1.24$ meter, $\sim 5 \%$ ). The localization performance shows a similar pattern as the standard deviation of the residuals $\left(X_{\sigma}\right)$ plotted in Figure 6. This is because the localization performance of MLE increases linearly with a decreasing value of the shadowing component (e.g. [10]).

- RAD significantly increases the performance as the number of calibration measurements increases (from $\sim 2.06$ to $\sim 1.25$ meter, $\sim 40 \%$ ). The localization performance show a similar pattern as the standard deviation of the residuals plotted in Figure 6. However, the localization performance still increases marginally with 48 or more calibration measurements (Figure 9), while the standard deviation of the residuals increases marginally (Figure 6). We believe that this increase originates from the fact that the plotted residuals are not completely independent.

Figure 9 shows that MLE outperforms ECO and RAD with a minimum number of calibration measurements (from $\sim 1.30$ to $\sim 1.76$ and $\sim 2.06$ meter). Moreover, RAD provides similar results as MLE and outperforms ECO with a maximum number of calibration measurements (from $\sim 1.25$ to $\sim 1.25$ and $\sim 1.76$ meter). Figure 9 shows that the "Ideal" performance of:

- ECO is worse than the "Realistic" performance (from $\sim 1.76$ to $\sim 2.04$ meter, $\sim 15 \%$ difference). We expect that this difference is caused by small RSS measurement deviations that influence the order of RSS measurements. This means that small RSS measurement deviations could cause relatively large performance differences.

- MLE is similar to the "Realistic" performance (1.25 and 1.23 meter), which is in accordance with the results presented in Section IV, Figure 6.

- RAD is significantly better than the "Realistic" performance, which is in accordance with the results presented in Section IV, Figure 6.

Table II provides an overview of the performance of the localization methods in relation to the following aspects:

- The Calibration measurements column represents to what extent the performance depends on the number of calibration measurements.

- The Accuracy column represents how well the localization method performs in environments with static sources of noise.

The "+"/“"-" represents how well the localization method scores $\mathrm{good} / \mathrm{bad}$ on this aspect.

\section{B. Number of Reference Nodes}

This subsection analyzes the localization performance as a function of the number of evaluated reference nodes, as the 


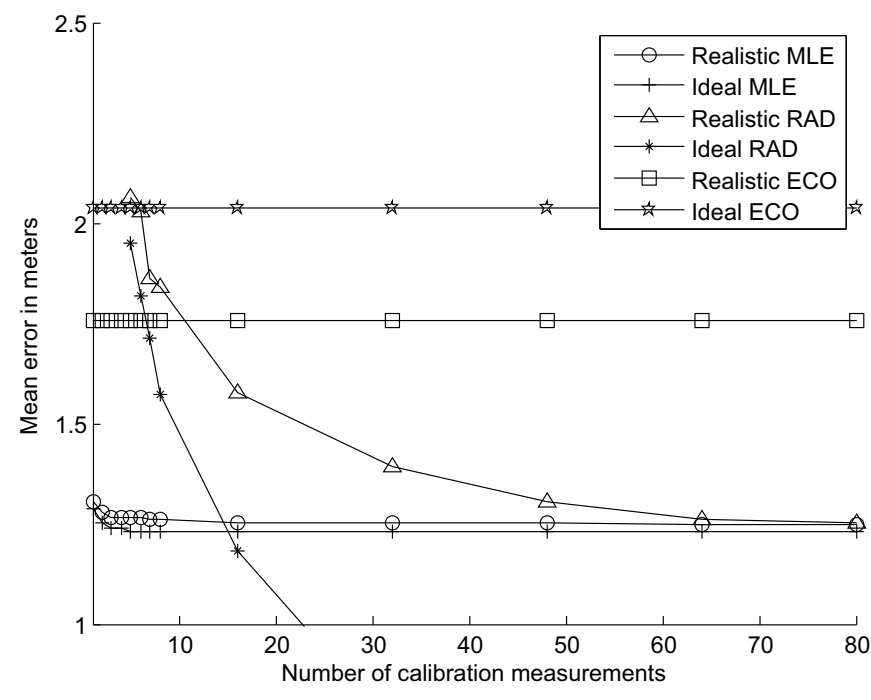

Fig. 9. Performance as a function of the number of calibration measurements

\begin{tabular}{|l|l|l|}
\hline methodology & Calibration measurements & Accuracy \\
\hline Fingerprinting & - & + \\
Range-based & + & + \\
Proximity & + & - \\
\hline
\end{tabular}

TABLE II

LOCALIZATION METHODOLOGIES

number of reference nodes increase the deployment costs. We change this number by altering the set of evaluated reference nodes. This basically means that there are multiple reference node set-ups per number of evaluated reference nodes. The mean performance of these reference node set-ups represents the performance per number of evaluated reference nodes. The mean error also provide insight to what extend the performance depends on the reference node set-up. Figure 10 shows the mean localization error as a function of the number of evaluated reference nodes. This figure shows that:

- The performance of MLE and RAD is significantly better than ECO. We believe that this increase originates from the fact that ECO is more sensitive to the reference node set-up.

- The performance of MLE is slightly better than RAD.

\section{Discussion Results}

Our results are based on line-of-sight measurements in a static environment. We did not consider environmental influences such as walls ([3]), antenna orientations ([27]), height of the radios ([23]), hardware differences between nodes ([7]) and moving objects ([3]). The main advantage of fingerprinting over proximity- and range-based localization algorithms is that it copes better with static sources of errors such as walls. However, real environments have dynamic sources of errors. Such dynamic error sources influence the performance of all localization methods described in this

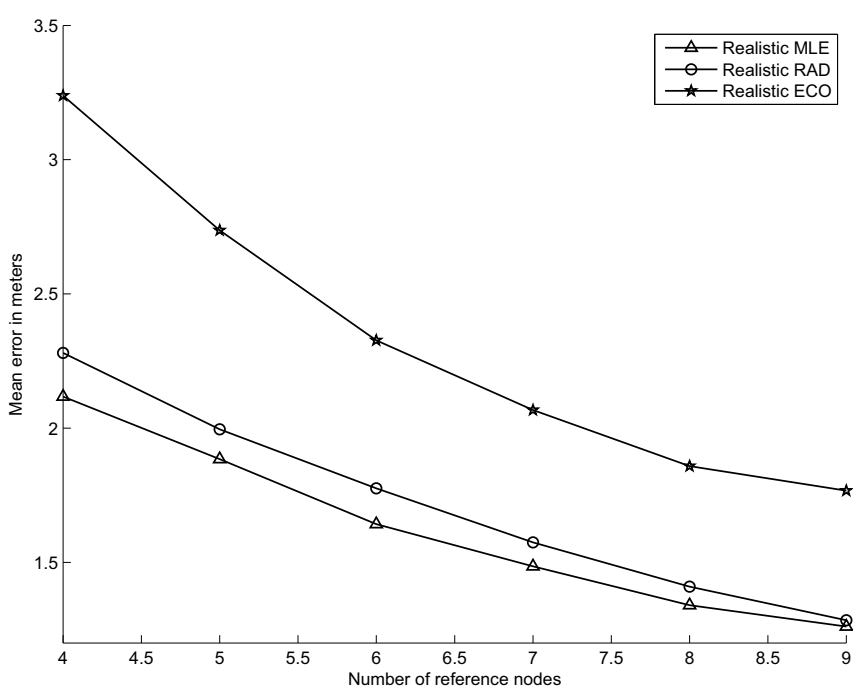

Fig. 10. Performance as a function of the number of reference nodes

paper. [7], [20], [23] and [27] all show that these dynamic sources of error are significant and that proper calibration of the propagation model is then required. The results in this paper show that fingerprinting-based localization algorithms require significantly more calibration effort to provide similar results as the range-based localization algorithms when they have to cope with dynamic sources of error.

\section{CONCLUSION}

In this study, we demonstrated that the influence of the number of calibration measurements on the localization performance differs per RSS-based method. We quantified this difference by performing an extensive amount of RSS measurements in an indoor environment. These measurements show that range-based localization algorithms outperform fingerprinting- and proximity-based localization algorithms. In addition, these measurements show that range-based localization algorithms are less susceptible to the difference between the calibration and localization measurements than the other localization methods. Therefore, we conclude that rangebased algorithms outperform fingerprinting- and proximitybased localization algorithms in terms of performance and applicability.

The analysis of the calibration phase of the different localization methods shows that the Log-Normal Shadowing model provides the best representation of the signal strength over the surface in a line-of-sight indoor environment. Therefore, it is not surprising that the range-based localization algorithm outperforms the other localization methods. However, this paper did not address the localization problem within an environment with static sources of error like walls. In our future research, we perform an extensive amount of RSS measurement in an office environment with walls and combine the strengths of range and fingerprint-based localization algorithms. 


\section{ACKNOWLEDGEMENTS}

This paper describes work in part undertaken in the context of the SENSEI project, "Integrating the Physical with the Digital World of the Network of the Future" (www.senseiproject.eu). SENSEI is a Large Scale Collaborative Project supported by the European 7th Framework Programme, contract number: 215923.

\section{REFERENCES}

[1] Hashemi H.: The indoor radio propagation channel, Proc. IEEE, July 1993, pp. 943- 996.

[2] Rappaport T.S., Wireless Communication: Principles and Practice, Prentice Hall, ISBN 013 3755633, 1996.

[3] P. Bahl and V. N. Padmanabhan: RADAR: An In-Building RF-Based User Location and Tracking System, in Proceedings of the 19th IEEE International Conference on Computer Communications (INFOCOM), March 2000.

[4] P.Bahl, V.N.Padmanabhan, A.Balachandran: Enhancements to the RADAR User Location and Tracking System, Microsoft Research Technical Report, February 2000.

[5] N.Patwari, R.J.O'Dea, Y.Wang: Relative Location in Wireless Networks. Presented at IEEE Vehicular Technology Conference, Spring, Rhodes, Greece, May 2001.

[6] D.Niculescu, B.Nath: Ad hoc positioning systems. In: IEEE Globecom 2001, San Antonio. 2001.

[7] K.Whitehouse, D.Culler: Calibration as Parameter Estimation in Sensor Networks. In ACM International Workshop on Wireless Sensor Networks and Applications, Atlanta, GA, USA, September 2002.

[8] T.He, C.Huang, B.M.Blum, J.Stankovic, T.Abdelzaher: Range-free localization schemes for large scale sensor networks. MobiCom, San Diego, California, September 2003, pp. 81-95.

[9] R.Nagpal, H.Shrobe, J.Bachrach: Organizing a Global Coordinate System from Local Information on an Ad Hoc Sensor Network. 2nd International Workshop on Information Processing in Sensor Networks (IPSN). April 2003.

[10] N. Patwari, A.O. Hero, M.Perkins, N.S.Correal, R.J.O'Dea: Relative Location Estimation in Wireless Sensor Networks. IEEE Transactions on Signal Processing, special issue on Signal Processing in Networks, vol. 51, no. 8, August 2003, pp. 2137-2148.

[11] K.Whitehouse, D.Culler: Macro-Calibration in Sensor/Actuator Networks. Mobile Networks and Applications Journal (MONET), Special Issue on Wireless Sensor Networks, ACM Press, June, 2003.

[12] P.Krishnan, A.S.Krishnakumar, W.Ju, C.Mallows, S.Ganu: A system for lease: Location estimation assisted by stationary emitters for indoor $\mathrm{rf}$ wireless networks. In Proceedings of the IEEE International Conference on Computer Communications (INFOCOM), Oct 2004.

[13] E.Elnahraway, X.Li, R.P.Martin: The limits of localization using rss. In SenSys 2004: Proceedings of the 2nd international conference on Embedded networked sensor systems, pages 283284, New York, NY, USA, 2004. ACM Press.

[14] C.Liu, K.Wu, T.He: Sensor localization with ring overlapping based on comparison of received signal strength indicator, in IEEE Mobile Ad-hoc and Sensor Systems (MASS), Oct. 2004

[15] K.Yedavalli, B.Krishnamachari, S.Ravula, and B.Srinivasan: Ecolocation: A sequence based technique for RF-only localization in wireless sensor networks. In IEEE IPSN 2005, April 2005.

[16] N.Patwari: Location estimation in sensor networks. Thesis of Neal Patwari at University of Michigan, 2005.

[17] J.A.Costa, N.Patwari, A.O.Hero: Distributed Weighted Multidimensional Scaling for Node Localization in Sensor Networks. ACM Transactions on Sensor Networks, Feb. 2006, vol. 2, no. 1, pp. 39-64.

[18] B.Li, J.Salter, A.G.Dempster, C.Rizos: Indoor positioning techniques based on wireless LAN, School of Surveying and Spatial Information Systems, UNSW, Sydney, Australia, Tech. Rep., 2006.

[19] Rong Peng, Mihail L. Sichitiu: Probabilistic Localization for Outdoor Wireless Sensor Networks. ACM SIGMOBILE Mobile Computing and Communications, Volume 11, Issue 1, January 2007, pp. 53-64.

[20] K.Whitehouse, C.Karlof, D.Culler: A Practical Evaluation of Radio Signal Strength for Ranging-based Localization. Mobile Computing and Communications Review, Volume 11, Number 1, 2007.
[21] R.A.Malaney: Nuisance Parameters and Location Accuracy in LogNormal Fading Models. IEEE Transactions on Wireless Communications, March 2007, Volume: 6, page(s): 937-947

[22] T.King, T.Haenselmann, W.Effelsberg: Deployment, Calibration, and Measurement Factors for Position Errors in 802.11-based Indoor Positioning Systems. In Proc. of the 3rd Int. Symp. on Location- and ContextAwareness (LoCA), Oberpfaffenhofen, Germany, Sept. 2007.

[23] T.Stoyanova, F.Kerasiotis, A.Prayatia and G.Papadopoulos: Evaluation of Impact Factors on RSS Accuracy for Localization and Tracking Applications. Proceedings of the 5th ACM international Workshop on Mobility Management and Wireless Access (Mobiwac), 2007.

[24] J.Shirahama and T.Ohtsuki: RSS-based localization in environments with different path loss exponent for each link. In IEEE Vehicular Technology Conference, 2008, pp. 1509-1513.

[25] V.Honkavirta, T.Perala, S.Ali-Loytty, R.Piche: A Comparative Survey of WLAN Location Fingerprinting Methods. Proc. of the 6th Workshop on Positioning, Navigation and Communication 2009 (WPNC'09), pages 243-251.

[26] B.J.Dil, P.J.M.Havinga: COM-LOC: A Distributed Range-Free Localization Algorithm in Wireless Networks. In: Proceedings of the 5th International Conference on Intelligent Sensors, Sensor Networks and Information Processing (ISSNIP), 7-12-2009, Mellbourne, Australia. pp. 457-462.

[27] B.J.Dil, P.J.M.Havinga: RSS-Based Localization with Different Antenna Orientations. Australian Telecommunication Networks and Applications Conference (ATNAC), 2010.

[28] http://focus.ti.com/lit/ds/symlink/cc2430.pdf, 2010. 